\title{
Fast and Green Method to Synthesis of Quinolone Carboxylic Acid Derivatives Using Giant-Ball Nanoporous Isopolyoxomolybdate as Highly Efficient Recyclable Catalyst in Refluxing Water
}

\author{
Yahya Mirzaie, ${ }^{1}$ Jalil Lari, ${ }^{1}$ Hooshang Vahedi, ${ }^{1}$ Mohammad Hakimi, ${ }^{1}$ Ahmad Nakhaei ${ }^{*}$ \\ and Abdolreza Rezaeifard ${ }^{3}$ \\ 1 Department of Chemistry, Payame Noor University, 19395-3697 Tehran, Iran \\ 2 Young Researchers and Elite Club, Mashhad Branch, Islamic Azad University, Mashhad, Iran \\ E-mail: nakhaei a@yahoo.com, nakhaei a@mshdiau.ac.ir \\ 3 Catalysis Research Laboratory, Department of Chemistry, Faculty of Science, University of Birjand, Birjand, $97179-414$ Iran
}

Received October 10th, 2016; Accepted January 11th, 2017.

\begin{abstract}
Various potentials antibacterial fluoroquinolone compounds were prepared by the direct amination of 7-halo-6- fluoroquinolone-3-carboxylic acids with variety of piperazine derivatives and $(4 \mathrm{aR}, 7 \mathrm{aR})$-octahydro-1H-pyrrolo[3,4-b] pyridine using $\left(\mathrm{NH}_{4}\right)_{42}$ $\left[\mathrm{Mo}^{\mathrm{VI}}{ }_{72} \mathrm{MoV}_{60} \mathrm{O}_{372}\left(\mathrm{CH}_{3} \mathrm{COO}\right)_{30}\left(\mathrm{H}_{2} \mathrm{O}\right)_{72}\right]$, a Keplerate-type giant-ball nanoporous isopolyoxomolybdate, as a catalyst in refluxing water. The results showed that this catalyst acts as effective catalyst and the reaction proceeded more easily and gave the highest yields of the products in short reaction time under refluxing water. Short reaction times, simple isolation of the products, and usage of eco-friendly catalysts are some features of this procedure. In addition, the catalysts was easily recovered and used in multiple catalytic cycles. This material was prepared according to a previously published literature procedure using inexpensive and readily available starting materials.
\end{abstract}

Keywords: Fluoroquinolone derivatives; fast and green synthesis; Keplerate $\left\{\mathrm{Mo}_{132}\right\}$.

\section{Introduction}

Fluoroquinolones have been a class of important of synthetic antibacterial agents which are widely used in clinic for the treatment of infectious diseases [1,2]. These compounds act with an excellent activity against gram-negative and comparatively moderate against gram-positive bacteria [3-7]. Mechanism of action of these compounds is based on inhibition of an enzyme essential for bacterial DNA replication called DNA gyrase [8]. It also appears that some fluoroquinolones possess anticancer and even anti-HIV activities [9-11].

Despite there are still certain undesired events in usage of fluoroquinolones for therapeutic purposes, fluoroquinolones are one of the most important antimicrobial agents with many advantages for clinical use. Therefore there has been a growing interest in the structure modification of the fluoroquinolone skeleton and in the development of its new derivatives with increasing efficacy to prevention of hospital-acquired infections induced by fluoroquinolone-resistant pathogens [12-14]. Recent studies have shown that substituents at the 7-position of the fluoroquinolone framework highly affect their biological
Resumen. Se prepararon varios derivados de la fluoroquinolona con actividad antibacterial potencial por aminación directa de ácidos 7-halo-6-fluoroquinolona-3-carboxílicos con derivados de la piperazina empleando como catalizador un isopolioxomolibdato nanoporoso "giant-ball" en agua a ebullición. Los resultados demuestran que este catalizador actúa como un catalizador efectivo y que la reacción procede más fácilmente y da mejores rendimientos bajo tiempos de reacción cortos en agua a ebullición. Algunas características importantes de este método son tiempos de reacción cortos, aislamiento simple de los productos y el empleo de catalizadores amigables con el ambiente. Además, el catalizador fue fácilmente recuperado y usado en múltiples ciclos catalíticos. El material fue preparado de acuerdo a un procedimiento previamente publicado empleando materiales baratos y disponibles comercialmente.

Palabras clave: Derivados de la flouroquinolona; síntesis rápida y verde; Keplerate $\left\{\mathrm{Mo}_{132}\right\}$.

activity, antimicrobial spectrum, strength and target preferences [15]. For example, piperazinyl moieties substitution at this position of fluoroquinolones increase their basicity, lipophilicity and their ability to penetrate into cell walls which leads to a wide range of clinically beneficial fluoroquinolone such as ciprofloxacin, enrfoloxacin, levofloxacin, etc. [16-18].

Many synthetic protocols have been developed to accelerate the rate of amination of fluoroquinolones and to improve the yield [19-29]. Major drawbacks of these procedures include expensive reagents, use of large amounts of toxic organic solvents, prolonged heating and side reactions or using microwave. These disadvantages are not acceptable in the current pharmaceutical industry. Therefore, the development of a new greener and more convenient method for the synthesis of fluoroquinolones is highly desirable.

Giant nanosized porous Keplerate-type POMs was reported for the first time by Müller and co-workers [30]. The Keplerate and giant nanosized porous POMs show unique features which can be considered as the basis of a new type of nanochemistry and nanomaterials science [31,32]. They find a large variety of applications in principal and applied science, such as 
in modelling passive cation transport through membranes, encapsulation, nanoseparation chemistry, and magnetic and optics properties [33,34].

According to the excellent acidic properties of solid polyoxometalate acids, in the last three decades, many applications as the useful and versatile acid catalysts have found in these structures [35]. Polyoxometalate acids are generally solids that are unsolvable in non-polar solvents but extremely soluble in polar ones and they can be used in both homogeneous and heterogeneous systems. Furthermore, these structures have a number of utilities involving powerful flexibility in qualification of the acid potency, easy handling, environmental friendly, non-toxicity and facile synthesis [36,37].

As a result of global interest in the ongoing research towards the development of environmentally friendly methods for the synthesis of organic compounds especially compounds that are frequently used in current pharmaceutical industry, we report herein facile and efficient green synthesis of fluoroquinolones as potential antibacterial with short reaction time by the two-component condensation of variety amines and some 7-halo-6-fluoroquinolone-3-carboxylic acids using a Keplerate-type giant-ball nanoporous isopolyoxomolybdate, $\left\{\mathrm{Mo}_{132}\right\}$, as a new catalyst with high catalytic activity under reflux condition in high yield.
In continuation of our previous works on the application of $\left(\mathrm{NH}_{4}\right)_{42}\left[\mathrm{Mo}^{\mathrm{VI}}{ }_{72} \mathrm{Mo}^{\mathrm{V}}{ }_{60} \mathrm{O}_{372}\left(\mathrm{CH}_{3} \mathrm{COO}\right)_{30}\left(\mathrm{H}_{2} \mathrm{O}\right)_{72}\right]$, a Keplerate-type giant-ball nanoporous isopolyoxomolybdate, represented as $\left\{\mathrm{Mo}_{132}\right\}$, as a catalyst for a series of organic transformations [38-40], we report here the application of this material as highly efficient and reusable novel catalyst to promote the reaction time, and yields of fluoroquinolone derivatives from the reaction of some 7-halo-6-fluoroquinolone carboxylic acid $\mathbf{1}$ and amine $\mathbf{2}$ in refluxing water under clean synthesis (Scheme 1). The diameter of this ball-shaped POM which calculated theoretically is $2.9 \mathrm{~nm}[31,32]$. For the first time this molybdenum cluster has been characterized by the TEM image by Polarz et al. [33]. The TEM picture clearly shows a periodic structure with an average size approximately $3 \mathrm{~nm}$ diameter. This experimentally obtained diameter fits nicely with the theoretical value for the inner diameter of the ball-shaped POM [31, 32].

\section{Results and discussion}

The $\left\{\mathrm{Mo}_{132}\right\}$ catalyst was characterized using FT-IR and UVvisible spectroscopies as reported in our previous work [38]. The catalytic activity of this material was evaluated in the
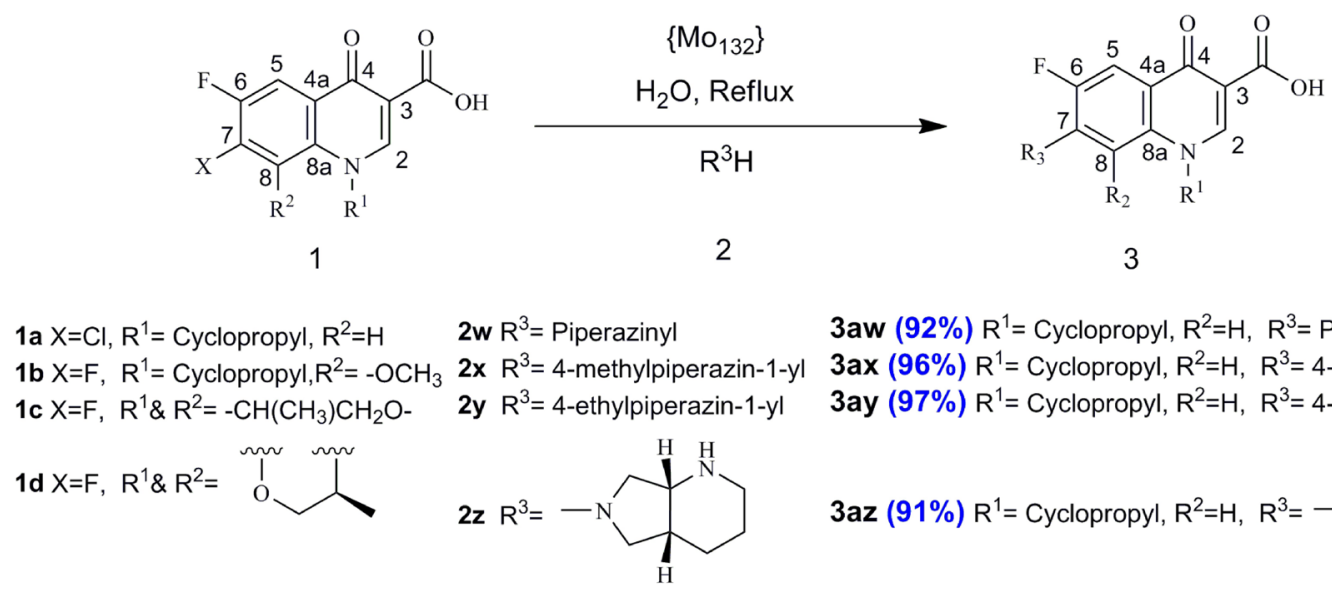

3aw $(92 \%) \mathrm{R}^{1}=$ Cyclopropyl, $\mathrm{R}^{2}=\mathrm{H}, \mathrm{R}^{3}=$ Piperazinyl

$3 a x(96 \%) R^{1}=$ Cyclopropyl, $R^{2}=H, R^{3}=4$-methylpiperazin-1-yl

3ay $(97 \%) R^{1}=$ Cyclopropyl, $R^{2}=H, R^{3}=4$-ethylpiperazin-1-yl

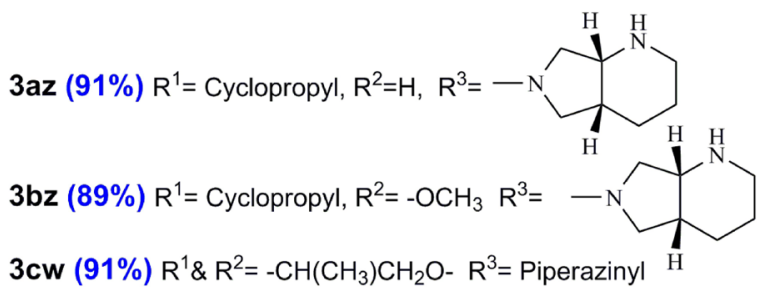

$3 \mathrm{cx}(89 \%) \mathrm{R}^{1} \& \mathrm{R}^{2}=-\mathrm{CH}\left(\mathrm{CH}_{3}\right) \mathrm{CH}_{2} \mathrm{O}-\mathrm{R}^{3}=4$-methylpiperazin-1-yl

$3 \mathrm{dw}(95 \%) \mathrm{R}^{1} \& \mathrm{R}^{2}=\stackrel{\text { O }}{\mathrm{O} \sim} \mathrm{R}^{3}=$ Piperazinyl

$3 d x(93 \%) R^{1} \& R^{2}=\stackrel{\sim}{O} \sim R^{3}=4$-methylpiperazin-1-yl

3dy $(88 \%) R^{1} \& R^{2}=\stackrel{2 \sim}{2} R^{3}=4$-ethylpiperazin-1-yl

$3 d z(94 \%) R^{1} \& R^{2}=\stackrel{O}{O}$

Scheme 1. Synthesis of fluoroquinolone derivatives in the presence of $\left\{\mathrm{Mo}_{132}\right\}$ under refluxing water. 
synthesis of fluoroquinolone derivatives. At first, the synthesis of compound 3ay was selected as a model reaction to determine suitable reaction conditions. The reaction was carried out by mixture of 7-chloro-1-cyclopropyl-6-fluoro-4-oxo-1,4-dihydroquinoline-3-carboxylic acid 1a $(1 \mathrm{mmol})$ and $N$-ethylpiperazine $2 \mathbf{y}(1.5 \mathrm{mmol})$ in the presence of different amounts of $\left\{\mathrm{Mo}_{132}\right\}$, and various solvents such as $\mathrm{EtOH}, \mathrm{H}_{2} \mathrm{O}, \mathrm{MeOH}$, $\mathrm{CH}_{3} \mathrm{CN}, \mathrm{CH}_{2} \mathrm{Cl}_{2}$, and also under solvent-free conditions at different temperature (Table 1). Long reaction times (>120 min) and not so good yields ( $45 \%$ ) of the product 3 ay were obtained in the absence of the catalyst in all cases (entries 1-5). On the other hand, different amounts of the catalyst $(0.02,0.04,0.06$, 0.08 , and 0.1 ) in the presence of the solvents or solvent-free condition in various temperatures caused to improve the yields and times of the reaction. Moreover, the best results in the presence of different amounts of catalyst were in refluxing solvents. These outcomes show that catalyst, solvent, and temperature are necessary for this reaction as well polar solvents were better than other non-polars. Also, the best yields and short reaction times were obtained in $0.08 \mathrm{~g}$ of the catalyst in water at different temperature (entries 12-14). Whereas, further increase in catalyst amount to $0.1 \mathrm{~g}$, did not improve the product yield and reaction time (entry 15). Among the tested solvents and also solvent-free conditions and various amounts of the catalyst, the reaction was more facile and proceeded to give the highest yield (97\%), and short reaction time (30 $\mathrm{min})$, using 0.08 $\mathrm{g}$ of $\left\{\mathrm{Mo}_{132}\right\}$ in $\mathrm{H}_{2} \mathrm{O}(5 \mathrm{ml})$ at reflux temperature (entry 12). All subsequent reactions were carried out in these optimized conditions.

According to these results, and in order to generalize this model reaction, we developed the reaction of $\mathbf{1 a - d}$ with a range of various amines $\mathbf{2} \mathbf{w}-\mathbf{z}$ under the optimized reaction conditions. The condensation of $\mathbf{1 a - d}$ and $\mathbf{2} \mathbf{w}-\mathbf{z}$ afforded the products 3 in high yields over relatively short reaction times in

Table 1. Optimization of reaction conditions for the synthesis of compound 3ay catalyzed by $\left\{\mathrm{Mo}_{132}\right\}$.

\begin{tabular}{|c|c|c|c|c|c|}
\hline Entry & Catalyst (g) & Solvent & $\mathrm{T} /{ }^{\circ} \mathrm{C}$ & Time/min & Isolated Yield $/ \%$ \\
\hline 1 & None & $\mathrm{EtOH}$ & Reflux & 125 & 29 \\
\hline 2 & None & $\mathrm{H}_{2} \mathrm{O}$ & Reflux & 125 & 42 \\
\hline 3 & None & $\mathrm{H}_{2} \mathrm{O}$ & r.t. & 160 & 34 \\
\hline 4 & None & Solvent-free & 100 & 150 & 16 \\
\hline 5 & None & Solvent-free & 120 & 150 & 19 \\
\hline 6 & 0.06 & Solvent-free & 120 & 100 & 27 \\
\hline 7 & 0.08 & Solvent-free & 120 & 100 & 31 \\
\hline 8 & 0.08 & Solvent-free & 100 & 100 & 25 \\
\hline 9 & 0.02 & $\mathrm{H}_{2} \mathrm{O}$ & Reflux & 80 & 59 \\
\hline 10 & 0.04 & $\mathrm{H}_{2} \mathrm{O}$ & Reflux & 60 & 76 \\
\hline 11 & 0.06 & $\mathrm{H}_{2} \mathrm{O}$ & Reflux & 40 & 88 \\
\hline 12 & 0.08 & $\mathrm{H}_{2} \mathrm{O}$ & Reflux & 30 & 97 \\
\hline 13 & 0.08 & $\mathrm{H}_{2} \mathrm{O}$ & 80 & 50 & 90 \\
\hline 14 & 0.08 & $\mathrm{H}_{2} \mathrm{O}$ & r.t. & 40 & 82 \\
\hline 15 & 0.1 & $\mathrm{H}_{2} \mathrm{O}$ & Reflux & 30 & 96 \\
\hline 16 & 0.04 & $\mathrm{EtOH}$ & Reflux & 70 & 58 \\
\hline 17 & 0.06 & $\mathrm{EtOH}$ & Reflux & 50 & 71 \\
\hline 18 & 0.08 & $\mathrm{EtOH}$ & Reflux & 45 & 86 \\
\hline 19 & 0.08 & $\mathrm{EtOH}$ & r.t. & 50 & 75 \\
\hline 20 & 0.06 & $\mathrm{MeOH}$ & Reflux & 60 & 63 \\
\hline 21 & 0.08 & $\mathrm{MeOH}$ & Reflux & 55 & 81 \\
\hline 22 & 0.08 & $\mathrm{MeOH}$ & r.t. & 60 & 74 \\
\hline 24 & 0.06 & $\mathrm{CH}_{3} \mathrm{CN}$ & Reflux & 70 & 48 \\
\hline 25 & 0.08 & $\mathrm{CH}_{3} \mathrm{CN}$ & Reflux & 65 & 78 \\
\hline 25 & 0.08 & $\mathrm{CH}_{3} \mathrm{CN}$ & r.t. & 70 & 67 \\
\hline 26 & 0.06 & $\mathrm{CH}_{2} \mathrm{Cl}_{2}$ & Reflux & 70 & 45 \\
\hline 27 & 0.08 & $\mathrm{CH}_{2} \mathrm{Cl}_{2}$ & Reflux & 60 & 57 \\
\hline 28 & 0.08 & $\mathrm{CH}_{2} \mathrm{Cl}_{2}$ & r.t. & 80 & 49 \\
\hline
\end{tabular}

* Reaction conditions: Ethyl 7-chloro-6-fluoroquinolone-3-carboxylic acids 1a (1 mmol) and $N$-ethylpiperazine $2 \mathbf{y}(1.5 \mathrm{mmol})$. 
Yahya Mirzaie et al.

refluxing water. The $\left\{\mathrm{Mo}_{132}\right\}$ efficiently catalyzed the reactions, giving the desired products in high yields over relatively short reaction times. Easy separation of obtained products from the catalyst makes this method useful for the synthesis of fluoroquinolones. Purity checks with melting points, TLC, HPLC $(>92 \%)$, and the ${ }^{1} \mathrm{H}$ NMR spectroscopic data reveal that only one product is formed in all cases and no undesirable side-products are observed. The structures of all known products $\mathbf{3}$ were deduced and compared with those of authentic samples from their melting points, ${ }^{1} \mathrm{H}$ NMR, ${ }^{13} \mathrm{C}$ NMR, and FT-IR spectral data [18-29].

To test the recyclability of $\left\{\mathrm{Mo}_{132}\right\}$, after completion of the model reaction, the catalyst was recovered according to the procedure described in the experimental section. The separated catalyst was dried at $60^{\circ} \mathrm{C}$ under vacuum for $1 \mathrm{~h}$ before being reused in the same reaction. The catalyst could be used at least five times without significant reduction in its activity $(97,96$, $94,94,93 \%$ yields in first to fifth use, respectively) which clearly demonstrates the practical reusability of this catalyst.

Although we did not investigate the reaction mechanism, on the basis of our previous reports [38-40], it is reasonable to assume that several accessible Mo sites and $\mathrm{NH}_{4}$ groups in $\left\{\mathrm{Mo}_{132}\right\}$ could act as Lewis acid and Brönsted acid centers, respectively, and therefore promote the necessary reactions. The catalyst would play a significant role in increasing the electrophilic character of the electrophiles in the reaction.

\section{Conclusion}

In conclusion, in this paper we developed the synthesis of fluoroquinolone derivatives $\mathbf{3 a w}, \mathbf{3 a x}, \mathbf{3 a z}, \mathbf{3 b z}, \mathbf{3 c w}, \mathbf{3 c x}, \mathbf{3 d w}$, $\mathbf{3 d x}, \mathbf{3 d y}$, and $\mathbf{3 d z}$ in the presence of $\left\{\mathrm{Mo}_{132}\right\}$, a Keplerate-type giant-ball nanoporous isopolyoxomolybdate, as a highly effective heterogeneous catalyst for the direct amination of 7-halo-6-fluoroquinolone-3-carboxylic acids 1a-d with several amines $\mathbf{2} \mathbf{w}-\mathbf{z}$ in refluxing water. This method provided these products in high yields over short reaction time, following a facile work-up process. The catalyst is inexpensive and easily obtained, stable and storable, easily recycled and reused for several cycles with consistent activity.

\section{Experimental}

\section{Chemicals and apparatus}

All chemicals were available commercially and used without additional purification. The catalyst was synthesized according to the literature [32]. Melting points were recorded using a Stuart SMP3 melting point apparatus. The FT-IR spectra of the products were obtained with $\mathrm{KBr}$ disks, using a Tensor 27 Bruker spectrophotometer. The ${ }^{1} \mathrm{H}$ NMR (300 MHZ) and ${ }^{13} \mathrm{C}$ NMR (75 MHZ) spectra were recorded using Bruker spectrometers.

\section{Typical procedure}

A mixture of 7-chloro-1-cyclopropyl-6-fluoro-4-oxo-1,4-dihydroquinoline-3-carboxylic acid $\mathbf{1 a}(1 \mathrm{mmol})$ and $N$-ethylpiperazine $2 \mathbf{y}(1.5 \mathrm{mmol})$ and $\left\{\mathrm{Mo}_{132}\right\}(0.08 \mathrm{~g})$ as catalyst in $\mathrm{H}_{2} \mathrm{O}(5$ $\mathrm{ml}$ ) was heated under reflux for the appropriate time. The reaction was monitored by TLC. Since the catalyst solubility is very high in cold water, after completion of the transformation, the reaction mixture was allowed to cool down into room temperature. The crude product was collected by filtration, washed with $\mathrm{H}_{2} \mathrm{O}$ and recrystallized from ethanol to give desired compounds 3ay. The catalyst could be readily recovered from the combined filtrate after evaporation to dryness under reduced pressure and washing with hot ethanol.

1-Cyclopropyl-6-fluoro-4-oxo-7-(piperazin-1-yl)-1,4-dihydroquinoline-3-carboxylic acid (3aw)

HPLC Purity: $99.14 \%$; Yield: $92 \%$; 20 min; m.p: $254-256{ }^{\circ} \mathrm{C}$ (lit. [23] 255-257 ${ }^{\circ} \mathrm{C}$ ); FT-IR ( $v \mathrm{~cm}^{-1} \mathrm{KBr}$ disc): 3533,3335 , 3033, 2912, 1705, 1623, 1494, 1447, 1383, 1271, 1144, 1024, 804; ${ }^{1} \mathrm{H}$ NMR (300 MHz, DMSO-d $\left.{ }_{6}\right): \delta 1.15-1.20(\mathrm{~m}, 2 \mathrm{H}$, $\left.\mathrm{CH}_{2}\right), 1.30-1.35\left(\mathrm{~m}, 2 \mathrm{H}, \mathrm{CH}_{2}\right), 2.90\left(\mathrm{t}, J=6.0 \mathrm{~Hz}, 4 \mathrm{H}, 2 \mathrm{CH}_{2}\right.$ ), $3.22\left(\mathrm{t}, J=6.0 \mathrm{~Hz}, 4 \mathrm{H}, 2 \mathrm{CH}_{2}\right), 3.75-3.85(\mathrm{~m}, 1 \mathrm{H}, \mathrm{CH}), 7.47(\mathrm{~d}$, $J=9.0 \mathrm{~Hz}, 1 \mathrm{H}, \mathrm{C} 8 \mathrm{H}), 7.75(\mathrm{~d}, J=15.0 \mathrm{~Hz}, 1 \mathrm{H}, \mathrm{C} 5 \mathrm{H}), 8.58$ (s, $1 \mathrm{H}, \mathrm{C} 2 \mathrm{H}) ;{ }^{13} \mathrm{C}$ NMR (75 MHz, DMSO-d 6 ): $7.9\left(\mathrm{CH}_{2}\right), 36.2$ $(\mathrm{NCH}), 45.8\left(2 \mathrm{NCH}_{2}\right), 51.1\left(2 \mathrm{NCH}_{2}\right), 106.9(\mathrm{C} 3), 107.1(\mathrm{C} 8)$, 111.4 (C5), 118.7 (C4a), 139.6 (C8a), 146.1 (C7), 148.2 (C2), 154.0 (C6), 165.6 (COOH), 176.6 (C4); Anal. Calc. for $\mathrm{C}_{17} \mathrm{H}-$ ${ }_{18} \mathrm{FN}_{3} \mathrm{O}_{3}(\%)$ : C, 61.62; H, 5.48; N, 12.68. Found: C, 61.54; H, $5.37 ; \mathrm{N}, 12.62$.

1-Cyclopropyl-6-fluoro-7-(4-methylpiperazin-1-yl)-4-oxo-1, 4-dihydroquinoline-3-carboxylic acid (3ax)

HPLC Purity: 97.92\%; Yield: 96\%; 25 min; m.p: $245-247{ }^{\circ} \mathrm{C}$ (lit. [22] 248-250 ${ }^{\circ} \mathrm{C}$ ); FT-IR ( $v \mathrm{~cm}^{-1} \mathrm{KBr}$ disc): 3428,3093 , $2935,1729,1626,1507,1469,1378,1299,1142,1007,885 ;{ }^{1} \mathrm{H}$ NMR (300 MHz, DMSO-d $)$ ): $\delta 1.17\left(\mathrm{~s}, 2 \mathrm{H}, \mathrm{CH}_{2}\right), 1.32(\mathrm{~d}, J=$ $\left.9.0 \mathrm{~Hz}, 2 \mathrm{H}, \mathrm{CH}_{2}\right), 2.23\left(\mathrm{~s}, 3 \mathrm{H}, \mathrm{NCH}_{3}\right), 2.20-2.35(\mathrm{~m}, 4 \mathrm{H}$, $\left.2 \mathrm{CH}_{2}\right), 3.00-3.10\left(\mathrm{~m}, 4 \mathrm{H}, 2 \mathrm{CH}_{2}\right), 3.75-3.85(\mathrm{~m}, 1 \mathrm{H}, \mathrm{CH}), 7.47$ $(\mathrm{d}, J=6.0 \mathrm{~Hz}, 1 \mathrm{H}, \mathrm{C} 8 \mathrm{H}), 7.75(\mathrm{~d}, J=12.0 \mathrm{~Hz}, 1 \mathrm{H}, \mathrm{C} 5 \mathrm{H}), 8.62$ (s, $1 \mathrm{H}, \mathrm{C} 2 \mathrm{H}) ;{ }^{13} \mathrm{C}$ NMR (75 MHz, DMSO-d 6 ): $8.0\left(2 \mathrm{CH}_{2}\right), 31.2$ $\left(\mathrm{NCH}_{3}\right), 36.3(\mathrm{NCH}), 45.9\left(2 \mathrm{NCH}_{2}\right), 49.4\left(2 \mathrm{NCH}_{2}\right), 106.0$ (C3), 107.1 (C8), 111.0 (C5), 118.0 (C4a), 139.6 (C8a), 146.1 (C7), 148.3 (C2), 151.0 (C6), 166.3 (COOH), 176.7 (C4); Anal. Calc. for $\mathrm{C}_{18} \mathrm{H}_{20} \mathrm{FN}_{3} \mathrm{O}_{3}(\%)$ : C, 62.60; H, 5.84; N, 12.17; Found: C, 62.53; H, 5.78; N, 12.11 .

1-Cyclopropyl-7-(4-ethylpiperazin-1-yl)-6-fluoro-4-oxo-1, 4-dihydroquinoline-3-carboxylic acid (3ay)

HPLC Purity: $99.06 \%$; Yield: 97\%; 30 min; m.p: $218-220{ }^{\circ} \mathrm{C}$ (lit. [22] 219-221 ${ }^{\circ} \mathrm{C}$ ); FT-IR ( $v \mathrm{~cm}^{-1} \mathrm{KBr}$ disc): 3533, 3335, 3033, 2912, 1738, 1627, 1470, 1381, 1337, 1254, 1154, 1022, 803; ${ }^{1} \mathrm{H}$ NMR (300 MHz, DMSO-d $\left.{ }_{6}\right): \delta 1.05(\mathrm{t}, J=7.0 \mathrm{~Hz}, 3 \mathrm{H}$, $\left.\mathrm{CH}_{3}\right), 1.10-1.35\left(\mathrm{~m}, 4 \mathrm{H}, 2 \mathrm{CH}_{2}\right), 2.42$ (q, $J=6.0 \mathrm{~Hz}, 2 \mathrm{H}, \mathrm{NCH}_{2}$ ), 2.50-2.60 (m, $8 \mathrm{H}, 4 \mathrm{CH}_{2}$, overlapped with solvent), 3.75-3.85 (m, 1H, CH), $7.55(\mathrm{~d}, J=6.0 \mathrm{~Hz}, 1 \mathrm{H}, \mathrm{C} 8 \mathrm{H}), 7.88(\mathrm{~d}, J=15.0$ 
$\mathrm{Hz}, 1 \mathrm{H}, \mathrm{C} 5 \mathrm{H}), 8.65$ (s, $1 \mathrm{H}, \mathrm{C} 2 \mathrm{H}), 15.23$ (s br., $1 \mathrm{H}, \mathrm{COOH}) ;{ }^{13} \mathrm{C}$ NMR (75 MHz, DMSO-d $\left.{ }_{6}\right): 8.0\left(2 \mathrm{CH}_{2}\right), 12.4\left(\mathrm{CH}_{3}\right), 36.2$ $(\mathrm{NCH}), 40.7\left(\mathrm{NCH}_{2}\right), 49.8-52.4\left(4 \mathrm{NCH}_{2}\right), 106.5(\mathrm{C} 3), 107.1$ (C8), 111.3 (C5), 118.8 (C4a), 139.5 (C8a), 145.5 (C7), 148.1 (C2), 155.0 (C6), 166.3 (COOH), 176.5 (C4); Anal. Calc. for $\mathrm{C}_{19} \mathrm{H}_{22} \mathrm{FN}_{3} \mathrm{O}_{3}$ (\%): C, 63.50; H, 6.17; N, 11.69; Found: C, $63.41 ; \mathrm{H}, 6.09 ; \mathrm{N}, 11.62$.

1-Cyclopropyl-6-fluoro-7-((4aR,7aR)-hexahydro-1H-pyrrolo [3,4-b]pyridin-6(2H)-yl)-4-oxo-1,4-dihydroquinoline-3-carboxylic acid (3az)

HPLC Purity: $92.96 \%$; Yield: $91 \%$; 18 min; m.p: $258-260{ }^{\circ} \mathrm{C}$ (lit. [24] 256-258 ${ }^{\circ} \mathrm{C}$ ); FT-IR ( $v \mathrm{~cm}^{-1} \mathrm{KBr}$ disc): 3504,3308 , 3076, 2938, 1719, 1629, 1549, 1509, 1412, 1336, 1180, 1108, $888 ;{ }^{1} \mathrm{H}$ NMR $\left(300 \mathrm{MHz}, \mathrm{DMSO}_{\mathrm{d}}\right.$ ): $\delta 1.10-1.35(\mathrm{~m}, 4 \mathrm{H}$, $\left.2 \mathrm{CH}_{2}\right), 1.55-1.70\left(\mathrm{~m}, 4 \mathrm{H}, 2 \mathrm{CH}_{2}\right), 2.50-2.60(\mathrm{~m}, 1 \mathrm{H}, \mathrm{CH}), 3.33$ (t, $\left.J=6.0 \mathrm{~Hz}, 2 \mathrm{H}, \mathrm{CH}_{2}\right), 3.30-3.55\left(\mathrm{~m}, 4 \mathrm{H}, 2 \mathrm{CH}_{2}\right), 3.63-3.75$ (m, H, CH), 6.91 (d, $J=6.0 \mathrm{~Hz}, 1 \mathrm{H}, \mathrm{C} 8 \mathrm{H}), 7.65(\mathrm{~d}, J=15.0 \mathrm{~Hz}$, $1 \mathrm{H}, \mathrm{C} 5 \mathrm{H}), 8.49(\mathrm{~s}, 1 \mathrm{H}, \mathrm{C} 2 \mathrm{H})$; Anal. Calc. for $\mathrm{C}_{20} \mathrm{H}_{22} \mathrm{FN}_{3} \mathrm{O}_{3}(\%)$ : C, 64.68; H, 5.97; N, 11.31; Found: C, 64.61; H, 5.59; N, 11.25.

1-Cyclopropyl-6-fluoro-7-((4aR,7aR)-hexahydro-1H-pyrrolo[3,4-b]pyridin-6(2H)-yl)-8-methoxy-4-oxo-1,4-dihydroquinoline-3-carboxylic acid (3bz)

HPLC Purity: $96.58 \%$; Yield: $89 \%$; 30 min, m.p: $239-241{ }^{\circ} \mathrm{C}$ (lit. [29] 238-242 ${ }^{\circ} \mathrm{C}$ ); FT-IR ( $v \mathrm{~cm}^{-1} \mathrm{KBr}$ disc): 3529,3470 , 3033, 2929, 1708, 1624, 1517, 1457, 1353, 1324, 1186, 1047, $805 ;{ }^{1} \mathrm{H}$ NMR $\left(300 \mathrm{MHz}\right.$, DMSO-d $\left.{ }_{6}\right): \delta 0.81-1.25(\mathrm{~m}, 4 \mathrm{H}$, $\left.2 \mathrm{CH}_{2}\right), 1.63-1.85\left(\mathrm{~m}, 4 \mathrm{H}, 2 \mathrm{CH}_{2}\right), 2.60-2.70\left(\mathrm{~m}, 2 \mathrm{H}, \mathrm{CH}_{2}\right)$, 3.10-3.20 (m, 1H, CH), $3.37\left(\mathrm{~s}, 3 \mathrm{H}, \mathrm{OCH}_{3}\right), 3.60-3.65(\mathrm{~m}, 1 \mathrm{H}$, $\mathrm{CH}), 3.70-3.80(\mathrm{~m}, 1 \mathrm{H}, \mathrm{CH}), 3.80-3.97\left(\mathrm{~m}, 2 \mathrm{H}, \mathrm{CH}_{2}\right), 4.04-$ $4.19\left(\mathrm{~m}, 2 \mathrm{H}, \mathrm{CH}_{2}\right), 7.63(\mathrm{dd}, J=12.0,3.0 \mathrm{~Hz}, 1 \mathrm{H}, \mathrm{C} 5 \mathrm{H}), 8.64$ (s, $1 \mathrm{H}, \mathrm{C} 2 \mathrm{H}), 15.15$ (s br., $\mathrm{COOH}) ;{ }^{13} \mathrm{C}$ NMR $(75 \mathrm{MHz}, \mathrm{DM}-$ SO-d $\left.{ }_{6}\right): 8.8\left(2 \mathrm{CH}_{2}\right) 10.0\left(\mathrm{CH}_{2}\right), 17.2\left(\mathrm{CH}_{2}\right), 20.9(\mathrm{CH}), 34.6$ $\left(\mathrm{NCH}_{2}\right), 39.1(\mathrm{NCH}), 41.1\left(\mathrm{NCH}_{2}\right), 41.8(\mathrm{NCH}), 54.4\left(\mathrm{NCH}_{2}\right)$, $62.3\left(\mathrm{OCH}_{3}\right), 106.8(\mathrm{C} 3), 117.6(\mathrm{C} 5), 134.9(\mathrm{C} 4 \mathrm{a}), 137.1(\mathrm{C} 8)$, 140.6 (C8a), 150.8 (C7), 151.7 (C2), 154.0 (C6), 166.3 $(\mathrm{COOH}), 176.4$ (C4); Anal. Calc. for $\mathrm{C}_{21} \mathrm{H}_{24} \mathrm{FN}_{3} \mathrm{O}_{4}$ (\%): C, $62.83 ;$ H, 6.03; N, 10.47; Found: C, 62.78; H, 5.94; N, 10.41.

9-Fluoro-3-methyl-7-oxo-10-(piperazin-1-yl)-3,7-dihydro2H-[1,4]oxazino[2,3,4-ij]quinoline-6-carboxylic acid (3cw) HPLC Purity: 95.19\%; Yield: 91\%; 27 min; m.p: $258-260{ }^{\circ} \mathrm{C}$ (lit. [27] 257-260 ${ }^{\circ} \mathrm{C}$ ); FT-IR ( $v \mathrm{~cm}^{-1} \mathrm{KBr}$ disc): 3255,3092 , $2968,1723,1573,1454,1392,1254,1023,1011,805 ;{ }^{1} \mathrm{H}$ NMR $\left(300 \mathrm{MHz}, \mathrm{DMSO}-\mathrm{d}_{6}\right): \delta 1.44\left(\mathrm{~d}, J=6.0 \mathrm{~Hz}, 3 \mathrm{H}, \mathrm{CH}_{3}\right), 2.80-$ $2.85\left(\mathrm{~m}, 4 \mathrm{H}, 2 \mathrm{CH}_{2}\right), 3.18-3.25\left(\mathrm{~m}, 4 \mathrm{H}, 2 \mathrm{CH}_{2}\right.$, overlapped with solvent), 4.37 (d, $J=12.0 \mathrm{~Hz}, 1 \mathrm{H}, \mathrm{CH}_{2}$ diastereotopic proton), 4.58 (d, $J=12.0 \mathrm{~Hz}, 1 \mathrm{H}, \mathrm{CH}_{2}$, diastereotopic proton), 4.85-4.95 (m, $1 \mathrm{H}, \mathrm{CH}), 7.51(\mathrm{dd}, J=12.0,6.0 \mathrm{~Hz}, 1 \mathrm{H}, \mathrm{C} 5 \mathrm{H}), 8.91(\mathrm{~s}, 1 \mathrm{H}$, $\mathrm{C} 2 \mathrm{H}) ;{ }^{13} \mathrm{C}$ NMR $\left(75 \mathrm{MHz}\right.$, DMSO-d 6 ): $18.4\left(\mathrm{CH}_{3}\right), 46.6$ $\left(2 \mathrm{NCH}_{2}\right), 52.0\left(2 \mathrm{NCH}_{2}\right), 55.2(\mathrm{NCH}), 68.4\left(\mathrm{OCH}_{2}\right), 103.6$ (C5), 107.1 (C3), 120.0 (C4a), 125.2 (C8a), 132.3 (C7), 140.5 (C8), 146.5 (C2), 154.0 (C6), 166.5 (COOH), 176.7 (C4); Anal. Calc. for $\mathrm{C}_{17} \mathrm{H}_{18} \mathrm{FN}_{3} \mathrm{O}_{4}(\%)$ : C, 58.78; H, 5.22; N, 12.10; Found: C, 58.72; H, 5.17; N, 10.36 .
9-Fluoro-3-methyl-10-(4-methylpiperazin-1-yl)-7-oxo-3,7-dihydro-2H-[1,4] oxazino[2,3,4-ij]quinoline-6-carboxylic acid (3cx) HPLC Purity: $99.89 \%$; Yield: $89 \%$; 20 min; m.p: $253-255^{\circ} \mathrm{C}$ (lit. [27] 250-257 ${ }^{\circ} \mathrm{C}$ ); FT-IR ( $v \mathrm{~cm}^{-1} \mathrm{KBr}$ disc): 3419,3335 , 3043, 2968, 1714, 1622, 1523, 1469, 1371, 1255, 1146, 1056, 804; ${ }^{1} \mathrm{H}$ NMR (300 MHz, DMSO-d 6 ): $\delta 1.44(\mathrm{~d}, J=9.0 \mathrm{~Hz}, 3 \mathrm{H}$, $\left.\mathrm{CH}_{3}\right), 2.22\left(\mathrm{~s}, 3 \mathrm{H}, \mathrm{NCH}_{3}\right), 2.35-2.50\left(\mathrm{~m}, 4 \mathrm{H}, 2 \mathrm{CH}_{2}\right), 3.20-3.40$ $\left(\mathrm{m}, 4 \mathrm{H}, 2 \mathrm{CH}_{2}\right), 4.35$ (dd, $J=12.0,3.0 \mathrm{~Hz}, 1 \mathrm{H}, \mathrm{CH}_{2}$, diastereotopic proton), $4.59\left(\mathrm{dd}, J=12.0,3.0,1 \mathrm{H}, \mathrm{CH}_{2}\right.$, diastereotopic proton), 4.85-4.98 (m, 1H, CH), $7.52(\mathrm{~d}, J=12.0 \mathrm{~Hz}, 1 \mathrm{H}, \mathrm{C} 5 \mathrm{H})$, 8.95 (s, 1H, C2H), 15.17 (s br., $1 \mathrm{H}, \mathrm{COOH}) ;{ }^{13} \mathrm{C}$ NMR $(75$ $\left.\mathrm{MHz}, \mathrm{DMSO}-\mathrm{d}_{6}\right): 18.4\left(\mathrm{CH}_{3}\right), 46.5\left(\mathrm{NCH}_{3}\right), 50.5\left(2 \mathrm{NCH}_{2}\right)$, $55.2\left(2 \mathrm{NCH}_{2}\right), 55.7(\mathrm{NCH}), 68.4\left(\mathrm{OCH}_{2}\right), 103.5(\mathrm{C} 5), 107.0$ (C3), 119.8 (C4a), 125.2 (C8a), 132.5 (C7), 140.5 (C8), 146.5 (C2), 154.2 (C6), 166.5 (COOH), 176.7 (C4); Anal. Calc. for $\mathrm{C}_{18} \mathrm{H}_{20} \mathrm{FN}_{3} \mathrm{O}_{4}$ (\%): C, 59.83; H, 5.58; N, 11.63; Found: C, 59.77; H, 5.08; N, 11.58 .

(S)-9-Fluoro-3-methyl-7-oxo-10-(piperazin-1-yl)-3,7-dihydro2H-[1,4]oxazino[2,3,4-ij]quinoline-6-carboxylic acid (3dw) HPLC Purity: $99.65 \%$; Yield: $95 \%$; 32 min; m.p: $260-262{ }^{\circ} \mathrm{C}$ (lit. [29] 263-265 ${ }^{\circ} \mathrm{C}$ ); FT-IR ( $v \mathrm{~cm}^{-1} \mathrm{KBr}$ disc): 3255, 3092, $2968,1723,1573,1454,1392,1254,1023,1011,805 ;{ }^{1} \mathrm{H}$ NMR (300 MHz, DMSO-d 6 ): $\delta 1.45$ (d, $J=6.0 \mathrm{~Hz}, 3 \mathrm{H}, \mathrm{CH}_{3}$ ), $2.75-$ $2.85\left(\mathrm{~m}, 4 \mathrm{H}, 2 \mathrm{CH}_{2}\right), 3.15-3.25\left(\mathrm{~m}, 4 \mathrm{H}, 2 \mathrm{CH}_{2}\right.$, overlapped with solvent), 4.30-4.40 (m, $1 \mathrm{H}, \mathrm{CH}_{2}$, diastereotopic proton), 4.52$4.62\left(\mathrm{~m}, 1 \mathrm{H}, \mathrm{CH}_{2}\right.$, diastereotopic proton), 4.85-4.95 $(\mathrm{m}, 1 \mathrm{H}$, $\mathrm{CH}), 7.51(\mathrm{~d}, J=12.0 \mathrm{~Hz}, 1 \mathrm{H}, \mathrm{C} 5 \mathrm{H}), 8.92(\mathrm{~s}, 1 \mathrm{H}, \mathrm{C} 2 \mathrm{H}) ;{ }^{13} \mathrm{C}$ NMR (75 MHz, DMSO-d $\left.{ }_{6}\right): 18.4\left(\mathrm{CH}_{3}\right), 45.8\left(2 \mathrm{NCH}_{2}\right), 51.0$ $\left(2 \mathrm{NCH}_{2}\right), 55.2(\mathrm{NCH}), 68.5\left(\mathrm{OCH}_{2}\right), 103.6(\mathrm{C} 5), 107.2(\mathrm{C} 3)$, 120.2 (C4a), 125.2 (C8a), 132.3 (C7), 140.5 (C8), 146.5 (C2), 154.2 (C6), $166.5(\mathrm{COOH}), 176.7$ (C4); Anal. Calc. for $\mathrm{C}_{17} \mathrm{H}-$ ${ }_{18} \mathrm{FN}_{3} \mathrm{O}_{4}(\%): \mathrm{C}, 58.78 ; \mathrm{H}, 5.22 ; \mathrm{N}, 12.10$; Found: C, 58.70; H, 4.93; N, 11.51 .

(S)-9-Fluoro-3-methyl-10-(4-methylpiperazin-1-yl)-7-oxo-3,7-dihydro-2H-[1,4]oxazino [2,3,4-ij]quinoline-6-carboxylic acid (3dx) HPLC Purity: $100 \%$; Yield: $93 \%$; 22 min; m.p: $225-227^{\circ} \mathrm{C}$ (lit. [25] 225-226 $\left.{ }^{\circ} \mathrm{C}\right)$; FT-IR ( $v \mathrm{~cm}^{-1} \mathrm{KBr}$ disc): $3251,3079,2973$, 1721, 1539, 1517, 1439, 1394, 1289, 1087, 1004, 801; ${ }^{1}$ H NMR $\left(300 \mathrm{MHz}, \mathrm{DMSO}-\mathrm{d}_{6}\right): \delta 1.44\left(\mathrm{~d}, J=6.0 \mathrm{~Hz}, 3 \mathrm{H}, \mathrm{CH}_{3}\right), 2.22(\mathrm{~s}$, $\left.3 \mathrm{H}, \mathrm{NCH}_{3}\right), 2.35-2.50\left(\mathrm{~m}, 4 \mathrm{H}, 2 \mathrm{CH}_{2}\right), 3.20-3.30\left(\mathrm{~m}, 4 \mathrm{H}, 2 \mathrm{CH}_{2}\right.$ ), 4.36 (dd, $J=12.0,3.0 \mathrm{~Hz}, 1 \mathrm{H}, \mathrm{CH}_{2}$, diastereotopic proton), 4.59 (dd, $J=12.0,3.0 \mathrm{~Hz}, 1 \mathrm{H}, \mathrm{CH}_{2}$, diastereotopic proton), $4.85-$ $4.95(\mathrm{~m}, 1 \mathrm{H}, \mathrm{CH}), 7.48(\mathrm{~d}, J=12.0 \mathrm{~Hz}, 1 \mathrm{H}, \mathrm{C} 5 \mathrm{H}), 8.94(\mathrm{~s}, 1 \mathrm{H}$, $\mathrm{C} 2 \mathrm{H}$ ), 15.15 (s br., $1 \mathrm{H}, \mathrm{COOH}$ ); ${ }^{13} \mathrm{C}$ NMR $(75 \mathrm{MHz}, \mathrm{DM}-$ $\left.\mathrm{SO}-\mathrm{d}_{6}\right): 18.4\left(\mathrm{CH}_{3}\right), 46.5\left(\mathrm{NCH}_{3}\right), 50.5\left(2 \mathrm{NCH}_{2}\right), 55.2\left(2 \mathrm{NCH}_{2}\right)$, $55.7(\mathrm{NCH}), 68.4\left(\mathrm{OCH}_{2}\right), 103.8(\mathrm{C} 5), 107(\mathrm{C} 3), 120(\mathrm{C} 4 \mathrm{a})$, 125.2 (C8a), 132.3 (C7), 140.4 (C8), 146.5 (C2), 154.2 (C6), $166.5(\mathrm{COOH}), 176.7$ (C4); Anal. Calc. for $\mathrm{C}_{18} \mathrm{H}_{20} \mathrm{FN}_{3} \mathrm{O}_{4}(\%)$ : C, 59.83; H, 5.58; N, 11.63; Found: C, 59.78; H, 5.50; N, 11.56 .

(S)-10-(4-Ethylpiperazin-1-yl)-9-fluoro-3-methyl-7-oxo-3,7-dihydro-2H-[1,4] oxazino[2,3,4-ij]quinoline-6-carboxylic acid (3dy) HPLC Purity: $99.26 \%$; Yield: 88\%; 20 min; m.p: $230-232{ }^{\circ} \mathrm{C}$ (lit. [26] 229-230 ${ }^{\circ} \mathrm{C}$ ); FT-IR ( $v \mathrm{~cm}^{-1} \mathrm{KBr}$ disc): 3432, 3042, 
$2975,1714,1623,1529,1478,1306,1243,1200,1010,743 ;{ }^{1} \mathrm{H}$ NMR (300 MHz, DMSO-d $\left.{ }_{6}\right): \delta 1.05\left(\mathrm{t}, J=6.0 \mathrm{~Hz}, 3 \mathrm{H}, \mathrm{CH}_{3}\right)$, 1.45 (d, $\left.J=9.0 \mathrm{~Hz}, 3 \mathrm{H}, \mathrm{CH}_{3}\right), 2.35-2.40$ (m, $2 \mathrm{H}, \mathrm{CH}_{2}$, overlapped with solvent) $2.40-2.60\left(\mathrm{~m}, 4 \mathrm{H}, 2 \mathrm{CH}_{2}\right), 3.15-3.20(\mathrm{~m}$, $\left.4 \mathrm{H}, 2 \mathrm{CH}_{2}\right), 4.37\left(\mathrm{~d}, J=12.0 \mathrm{~Hz}, 1 \mathrm{H}, \mathrm{CH}_{2}\right.$, diastereotopic proton), 4.57 (d, $J=9.0 \mathrm{~Hz}, 1 \mathrm{H}, \mathrm{CH}_{2}$, diastereotopic proton), 4.91 (d, $1 \mathrm{H}, J=6.0 \mathrm{~Hz}, \mathrm{CH}), 7.56$ (d, $J=12.0 \mathrm{~Hz}, 1 \mathrm{H}, \mathrm{C} 5 \mathrm{H}), 8.94$ (s, 1H, C2H); ${ }^{13} \mathrm{C}$ NMR (75 MHz, DMSO-d 6$)$ : $12.2\left(\mathrm{CH}_{3}\right), 18.4$ $\left(\mathrm{CH}_{3}\right), 46.5\left(\mathrm{NCH}_{2}\right), 50.5\left(2 \mathrm{NCH}_{2}\right), 53.4\left(2 \mathrm{NCH}_{2}\right), 55.3$ $(\mathrm{NCH}), 68.5\left(\mathrm{OCH}_{2}\right), 103.0(\mathrm{C} 5), 107.0(\mathrm{C} 3), 125.2(\mathrm{C} 4 \mathrm{a})$, 126.8 (C8a), 132.3 (C7), 140.0 (C8), 146.7 (C2), 154.0 (C6), $166.5(\mathrm{COOH}), 176.6(\mathrm{C} 4)$; Anal. Calc. for $\mathrm{C}_{19} \mathrm{H}_{22} \mathrm{FN}_{3} \mathrm{O}_{4}(\%)$ : C, 60.79; H, 5.91; N, 11.19; Found: C, 60.72; H, 5.84; N, 11.11.

(S)-9-Fluoro-10-((4aR,7aR)-hexahydro-1H-pyrrolo[3,4-b]pyridin-6(2H)-yl)-3-methyl-7-oxo-3,7-dihydro-2H-[1,4]oxazino[2,3,4-ij]quinoline-6-carboxylic acid (3dz)

HPLC Purity: 98.63\%; Yield: $94 \%$; 25 min; m.p: $265-267{ }^{\circ} \mathrm{C}$ (lit. [24] 265-268 $\left.{ }^{\circ} \mathrm{C}\right)$; FT-IR ( $v \mathrm{~cm}^{-1} \mathrm{KBr}$ disc): 3319,3044 , 2932, 1719, 1622, 1527, 1472, 1357, 1191, 1087, 1045, 862; ${ }^{1} \mathrm{H}$ NMR (300 MHz, DMSO-d $\left.\mathrm{d}_{6}\right): \delta 1.30-1.70\left(\mathrm{~m}, 4 \mathrm{H}, 2 \mathrm{CH}_{2}\right), 1.45$ $\left(\mathrm{d}, J=6.0 \mathrm{~Hz}, 3 \mathrm{H}, \mathrm{CH}_{3}\right), 2.10-2.20(\mathrm{~m}, 1 \mathrm{H}, \mathrm{CH}), 2.80-2.90(\mathrm{~m}$, $1 \mathrm{H}, \mathrm{CH}), 3.15-3.40\left(\mathrm{~m}, 4 \mathrm{H}, 2 \mathrm{CH}_{2}\right), 4.00-4.15\left(\mathrm{~m}, 2 \mathrm{H}, \mathrm{CH}_{2}\right)$, $4.23\left(\mathrm{~d}, J=12.0 \mathrm{~Hz}, 1 \mathrm{H}, \mathrm{CH}_{2}\right.$, diastereotopic proton), 4.59 (d, $J$ $=12.0 \mathrm{~Hz}, 1 \mathrm{H}, \mathrm{CH}_{2}$, diastereotopic proton), $4.80-4.92(\mathrm{~m}, 1 \mathrm{H}$, $\mathrm{CH}), 7.47(\mathrm{~d}, J=15 \mathrm{~Hz}, 1 \mathrm{H}, \mathrm{C} 5 \mathrm{H}), 8.85(\mathrm{~s}, 1 \mathrm{H}, \mathrm{C} 2 \mathrm{H})$; Anal. Calc. for $\mathrm{C}_{20} \mathrm{H}_{22} \mathrm{FN}_{3} \mathrm{O}_{4}(\%)$ : C, 62.01; H, 5.72; N, 10.85; Found: C, 61.96; H, 5.74; N, 10.78 .

\section{Acknowledgements}

We acknowledge the financial support from Payame noor University of Mashhad, Islamic Azad University, Mashhad Branch, and TEMAD Pharmaceutical Ingredients Company in Iran for their support.

\section{References}

1. Fernandes, P. B.; Shipkowitz, N.; Bower, R. R.; Jarvis, K. P.; Weisz, J.; Chu, D. T. J. Antimicrob. Chemother 1986, 18, 693-701.

2. Stein, G. E.; Goldstein, E. J. Clin. Infect. Dis. 2006, 42, 1598-1607.

3. Chen, Y. L.; Fang, K. C.; Sheu, J. Y.; Hsu, S. L.; Tzeng, C. C. J. Med. Chem. 2001, 44, 2374-2377.

4. Fujimaki, K.; Noumi, T.; Saikawa, I.; Inoue, M.; Mitsuhashi, S. Antimicrob. Agents Chemother. 1988, 32, 827-833.

5. Golet, E. M.; Strehler, A.; Alder, A. C.; Giger, W. Anal. Chem. 2002, 74, 5455-5462.

6. O'Donnell, J. A.; Gelone, S. P. Infect. Dis. Clin. North Am. 2000, 14, 489-513.

7. Zhanel, G. G.; Walkty, A.; Vercaigne, L.; Karlowsky, J. A.; Embil, J.; Gin, A. S.; Hoban, D. J. Can. J. Infect. Dis. Med. Microbiol. 1999, 10, 207-238.

8. Llorente, B.; Leclerc, F.; Cedergren, R. Bioorg. Med. Chem. 1996, 4, 61-71.
9. Wentland, M. P.; Lesher, G. Y.; Reuman, M.; Gruett, M. D.; Singh, B.; Aldous, S. C.; Dorff, P. H.; Rake, J. B.; Coughlin, S. A. J. Med. Chem. 1993, 36, 2801-2809.

10. Elsea, S. H.; Osheroff, N.; Nitiss, J. L. J. Biol. Chem. 1992, 267, 13150-13153.

11. Oh, Y. S.; Lee, C. W.; Chung, Y. H.; Yoon, S. J.; Cho, S. H. J. Heterocycl. Chem. 1998, 35, 541-550.

12. Karlowsky, J. A.; Adam, H. J.; Desjardins, M.; Lagacé-Wiens, P. R.; Hoban, D. J.; Zhanel, G. G.; Baxter, M. R.; Nichol, K. A.; Walkty, A.; Alliance, C. A. R. J. Antimicrob. Chemother. 2013, 68, i39-i46.

13. Gootz, T. D.; Brighty, K. E. Med. Res. Rev. 1996, 16, 433-486.

14. Aubry, A.; Pan, X. S.; Fisher, L. M.; Jarlier, V.; Cambau, E. Antimicrob. Agents Chemother. 2004, 48, 1281-1288.

15. Mitscher, L. A. Chem. Rev. 2005, 105, 559-592.

16. Sriram, D.; Aubry, A.; Yogeeswari, P.; Fisher, L. Bioorg. Med. Chem. Lett. 2006, 16, 2982-2985.

17. Dubar, F.; Anquetin, G.; Pradines, B.; Dive, D.; Khalife, J.; Biot, C. J. Med. Chem. 2009, 52, 7954-7957.

18. Shindikar, A. V.; Viswanathan, C. Bioorg. Med. Chem. Lett. 2005, 15, 1803-1806.

19. Reddy, P. G.; Baskaran, S. Tetrahedron Lett. 2001, 42, 6775-6777.

20. Kawakami, K.; Namba, K.; Tanaka, M.; Matsuhashi, N.; Sato, K.; Takemura, M. Antimicrob. Agents Chemother. 2000, 44, 2126-2129.

21. Schwarcz, R.; Kajii, Y.; Ono, S. I.; Ono S. I. U.S. Patent: 12/742171, 2008.

22. Grohe, K.; Heitzer, H. Liebigs Ann. Chem. 1987, 1987, 29-37.

23. Petersen, U.; Grohe, K.; Kuck, K. H. U.S. Patent: 4563459, 1986.

24. Petersen, U.; Schrock, W.; Habich, D.; Krebs, A.; Schenke, T.; Philipps, T.; Grohe, K.; Endermann, R.; Bremm, K. D.; Metzger, K. G. U.S. Patent: 5480879, 1996.

25. Lee, T. A. Khoo, J. H. Song S. H. Patent: WO2006009374, 2006.

26. Hayakawa, I.; Atarashi, S.; Imamura, M.; Yokohama, S.; Higashihashi, N.; Sakano, K.; Ohshima, M. U.S. Patent: 4985557, 1991.

27. Hayakawa, I.; Hiramitsu, T.; Tanaka, Y. Chem. Pharm. Bull. 1984, 32, 4907-4913.

28. Moxifloxacin. Tuberculosis, 2008, 88, 127-131.

29. Guruswamy, B.; Arul, R. Lett. Drug Des. Discov. 2013, 10, 86-93.

30. Müller, A.; Das, S. K.; Talismanov, S.; Roy, S.; Beckmann, E.; Bögge, H.; Schmidtmann, M.; Merca, A.; Berkle, A.; Allouche, L.; Zhou, Y. Angew. Chem. 2003, 115, 5193-5198.

31. Zhang, L.; Xiong, T.; Zhou, Y.; Zhang, L. Chem. Asian J. 2010, 5, 1984-1987.

32. Müller, A.; Krickemeyer, E.; Bögge, H.; Schmidtmann, M.; Peters, F. Angew. Chem. Int. Ed. 1998, 37, 3359-3363.

33. Polarz, S.; Smarsly, B.; Göltner, C.; Antonietti, M. Adv. Mater. 2000, 12, 1503-1507.

34. Greedan, J. E. J. Mater. Chem. 2001, 11, 37-53.

35. Kozhevnikov, I. V. Chem. Rev. 1998, 98, 171-198.

36. Kozhhevnikov, I. V. (Ed.), Catalysis for Fine Chemical Synthesis, Catalysis by Polyoxometalates 2, Wiley, New York, 2002.

37. Romanelli, G. P.; Bennardi, D.; Ruiz, D. M.; Baronetti, G.; Thomas, H. J.; Autino, J. C. Tetrahedron Lett. 2004, 45, 8935-8939.

38. Nakhaei, A.; Davoodnia, A. Chin. J. Catal. 2014, 35, 1761-1767.

39. Nakhaei, A.; Davoodnia, A.; Morsali, A. Res. Chem. Intermed. 2015, 41, 7815-7826.

40. Davoodnia, A.; Nakhaei, A. Synth. React. Inorg. M. 2016, 46, 1073-1080. 